\title{
Encoding specificity in the cued and free recall of categorically and alphabetically organized words*
}

\author{
PATRICIA A. LAUER $\dagger$ \\ University of Colorado, Boulder, Colorado 80302
}

\begin{abstract}
Sixty Ss learned a 40-word list comprised of five words from each of eight taxonomic categories and beginning with each of eight initial letters. Words were presented for study either cued and blocked by initial letter or by taxonomic category or randomly presented without cues. Output was cued by initial letters, taxonomic categories, or neither, followed by a second uncued recall test for all groups. Consistency of input organization and output cue facilitated both cued and uncued recall, but produced a decrement in cued relative to uncued recall. With output cues inconsistent with input organization, however, uncued recall was higher than cued recall. Clustering on the uncued free recall was dependent on input organization and was positively correlated with recall if categoric, but negatively correlated if alphabetic. Results were interpreted as supportive of an encoding specificity hypothesis.
\end{abstract}

Thomson and Tulving (1970) reported three experiments investigating their encoding specificity principle using associates of to-be-remembered (TBR) words as cues. Their first experiment demonstrated that weak cues could not facilitate recall of TBR words unless these cues were previously presented and presumably encoded with the words. Contrary to a strict view of encoding specificity, strong cues facilitated the recall of words previously presented with weak cues, although not as much as with strong or no previous cues.

Lauer and Battig (1972) demonstrated encoding specificity in multitrial free recall, using taxonomic category labels and initial letters as cues. Moreover, if words had been organized alphabetically as well as cued by initial letter, their Ss recalled slightly fewer words when switched to a category-cued test trial as compared with previous letter-cued recall, and showed much poorer category-cued recall than groups trained with category cues or with no cues. In addition, they found a marked superiority of the taxonomic over the letter cue in all phases of learning and recall.

These results suggested a replication of the critical conditions of the Thompson-Tulving (1970) Experiment I, but with taxonomic category labels as strong cues and initial letters as weak cues instead of strong and weak associates, and with appropriate blocking during input of lists with category and letter input cues to maximize Ss' usage of the appropriate cue type.

*This is publication No. 47 of the Institute for the Study of Intellectual Behavior and was partially supported by Grant GB-34077X from the National Science Foundation. The author gratefully acknowledges W. F. Battig for his assistance and W. Kintsch for his suggestion leading to the experiment's design. The author ran this experiment while she was an instructor at Austin College, Sherman, Texas. The paper is sponsored by W. F. Battig, who takes full editorial sponsored by $W$. $F$. Bat
responsibility for its contents.

+ Requests for reprints should be sent to Patricia A. Lauer Department of Psychology, University of Colorado, Boulder, Colorad o 80302 .

\section{METHOD}

\section{Design and Subjects}

The design was a 3 by 3 factorial manipulating input organization (categoric, alphabetic, or random) and output cue (taxonomic, letter, or none). The Ss were 60 Austin College introductory psychology students, all tested in a single classroom session which lasted $12 \mathrm{~min}$. Experiment test booklets were randomly assigned to the $60 \mathrm{Ss}$, with the restriction that each of the nine experimental conditions was represented once within each stack of nine booklets. Thus, there were seven Ss in six conditions and six Ss in the remaining three conditions (alphabetic input with taxonomic and uncued output, and categoric input with let ter output).

\section{Materials and Procedure}

Lauer and Battig's (1972) 40-word list, which was used for all nine experimental conditions, contained five instances of each of eight taxonomic categories (Battig \& Montague, 1969), as well as subsets of five words beginning with each of eight different letters. For categoric input conditions, the 40 words were typed in capital letters on a study sheet in a single column of eight groups, each containing the five instances of one of the taxonomic categories, with the category name typed in small letters and underlined at the top of each group. Under alphabetic input conditions, all Ss received study sheets with the 40 words presented in eight groups, each containing the five words beginning with each initial letter, the latter being capitalized and underlined at the top of each group. For both input conditions, the eight groups were randomly listed such that no two adjacent words ever belonged to the same alternative grouping not represented in input. For the random input group, the 40 words were randomly listed in a continuous column on the study sheet, with the restriction that no two adjacent words ever shared the same initial letter or taxonomic category.

On the first recall trial, cued output groups received a recall sheet on which each of the eight cues (taxonomic category label or initial letter) was typed and followed by five numbered lines. The uncued output group received a recall sheet with 40 numbered and ungrouped lines. This same uncued recall sheet was used by all groups on the second (uncued) recall trial.

Each $S$ received a 6-page test booklet, in which the first page contained instructions specifying that Ss would have $3 \mathrm{~min}$ to memorize 40 words which were typed in capitals on a study sheet in the booklet. All Ss were instructed that, for some of them, the 40 words would be separated into eight groups, with 
each group associated with the cue item which preceded it, while for others the words were simply listed on the sheet without any cue items. They were told nothing of the nature of the cues. After reading these instructions, Ss turned to the word list on Page 2 for a 3 -min study trial. They then had $50 \mathrm{sec}$ to read Page 3, which contained instructions about the recall sheet to follow. Those Ss in cued output conditions were told to list under each cue the five words they remembered being associated with that cue item, while uncued Ss were told to recall the words in any order that occurred to them. After $3 \mathrm{~min}$ for written cued recall on Page 4, Ss had $50 \mathrm{sec}$ to read Page 5, which was an instruction sheet concerning the 3 -min uncued recall that followed for all Ss on Page 6.

\section{RESULTS}

In general, taxonomic category organization was expected to produce higher word recall than organization based on initial letters. However, within each input condition, the output cue predicted to be most effective was that which was consistent with input encoding. These expectations were strongly confirmed, particularly by the cued recall means shown for the nine experimental conditions in Table 1.

Categoric input produced significantly higher recall than random input, which in turn showed significantly higher recall than alphabetic input $[F s(1,51) \geqslant 5.47$, $\mathrm{p}<.025]$. While taxonomic and uncued output groups did not differ $(F<1)$, both recalled significantly more words than with letter cues at output $[\mathrm{F}(1,51)=8.37$, $\mathrm{p}<.01]$.

Table 1 also shows that, with both categoric and random input conditions, taxonomic and no output cues produced much higher cued recall than did letter output cues. With alphabetic input, however, cued recall was higher with letters than with either taxonomic or no output cues. Thus, the interaction component contrasting letter vs taxonomic and uncued output as a function of alphabetic vs categoric and random input was highly significant $[F(1,51)=8.52, p<.01]$.

Uncued recall scores (bottom half of Table 1) generally corroborated the cued recall results described above, reflecting the high within-S correlation $(r=.85)$ between the two measures. The superiority of taxonomic and none over letter output cues only within categoric

Table 1 Mean Cued and Uncued Recall

\begin{tabular}{lcccc}
\hline & \multicolumn{4}{c}{ Input } \\
\cline { 2 - 5 } Output & Categoric & Alphabetic & Random & Total \\
\hline \multicolumn{5}{c}{ Cued Recall } \\
Taxonomic & 25.71 & 16.00 & 22.14 & 21.28 \\
Letter & 18.00 & 17.14 & 15.43 & 16.86 \\
None & 25.57 & 14.00 & 20.86 & 20.14 \\
Total & 23.09 & 15.71 & 19.48 & \\
& \multicolumn{5}{c}{ Uncued Recall } \\
Taxonomic & 23.71 & 17.00 & 20.00 & 20.24 \\
Letter & 20.83 & 16.71 & 16.00 & 17.85 \\
None & 27.14 & 15.00 & 21.71 & 21.28 \\
Total & 23.89 & 16.24 & 19.24 & \\
\hline
\end{tabular}

and random but not alphabetic input conditions, however, was sufficiently reduced that its interaction component did not approach significance $[F(1,51)=2.72, p>.10]$. As indicated by an analysis of difference scores between cued and uncued recall for each $\mathrm{S}$, this reflected in part decrements from cued to uncued recall, especially following taxonomic output cues with categoric $(-2.00)$ or random $(-2.14)$ input, but also for the alphabetic-input letter-output group $(-0.43)$, whereas all other groups showed increases from cued to uncued recall, especially the categoric-input letter-output group (2.83). Thus the cued-uncued difference scores were significantly poorer for taxonomic $(-1.04)$ than letter $(.99)$ or no output (1.14) cue conditions $[\mathrm{F}(1,51)=5.46, \mathrm{p}<.025]$, and the difference between these scores for categoric and random, as contrasted with alphabetic, input conditions was significantly opposite in direction for taxonomic as contrasted with letter output conditions $[F(1,51)=5.20, p<.05]$

To further assess the relative influence of input organization and output cue on cued recall, total recall was partitioned into number of categories recalled (NC) and items recalled per category (IPC) both for taxonomic and letter categories. For taxonomic NC recall, the only significant effects were those produced by type of output cue. Ss cued by taxonomic category labels (7.40) recalled significantly more taxonomic categories than did uncued Ss (6.75), and both recalled significantly more taxonomic categories than with letter output $(6.45)[\mathrm{Fs}(1,51) \geqslant 4.26, \mathrm{p}<.05]$. Results for items recalled per taxonomic category were the same as those for total cued recall, although the effects were more highly significant. As in the recall analysis, input accounted for a far greater proportion of the between-groups IPC variance $(80 \%)$ than did output $(10 \%)$. The only significant effect in the letter NC and IPC analysis reflected lower NC scores for uncued (5.67) than cued (6.71) output only following alphabetic input, whereas cued and uncued output were virtually identical for categoric and random input $[F(1,51)=6.00$, $\mathrm{p}<.025]$.

Analyses were also carried out for categoric and alphabetic ratio-of-repetition or clustering scores (observed/maximum) calculated for the uncued recall of each S. Separate analyses of categoric and alphabetic clustering and scores based on their differences indicated that input was the critical factor. Categoric input (.96) produced significantly more categoric clustering than random input $(.78)[F(1,51)=5.67, p<.025]$, and both showed much more categoric clustering than alphabetic input $(.42) \quad[\mathrm{F}(1,51)=44.50, \mathrm{p}<.001]$. Categoric (.03) and random (.11) input groups did not differ in alphabetic clustering but both were significantly below the alphabetic (.58) input group $[F(1,51)=84.75, \mathrm{p}<.001]$. A significant component of the input-output interaction indicated that letter cues produced more alphabetic clustering (.13) than no cues (.02) in taxonomic and random input groups, while with 
alphabetic input no cues (.81) were superior to letter cues $(.54)$ in alphabetic clustering $[F(1,51)=7.83$, $\mathrm{p}<.01]$.

The analysis of categoric-alphabetic clustering difference scores indicated significantly more overall categoric (.73) than alphabetic clustering (.23) $[F(1,51)=91.75, p<.001]$. The greatest differences favoring categoric clustering were observed in the categoric input group (.93), which significantly differed from random input $(.67)[F(1,51)=4.44, p<.05]$, with alphabetic input actually producing more alphabetic than categoric clustering $(-.16)$. Correlations between categoric and alphabetic clustering and uncued recall were .46 and -.50 , respectively, both $\mathrm{p}<.01$.

\section{DISCUSSION}

Encoding specificity was demonstrated on the cued recall test by the interaction of input with output. Within each output condition, the highest recall occurred for the group given the same cues as were used for encoding. The main focus of this pattern was the letter output, which produced large cued recall decrements in categoric and random input groups but a slight facilitation in the alphabetic input condition. In addition, the only groups which experienced a recall loss on the uncued test were those conditions whose previous output cue had been consistent with their input organization.

Contrary to Thomson and Tulving's (1970) results, facilitation by strong cues compared to no cues was very slight in both strong and random input groups, suggesting that, with categorized word lists, spontaneous reproduction of taxonomic labels is likely, given appropriate encoding. With weak or alphabetic input, strong output cues again showed a slight superiority to no cues. As suggested by Thomson and Tulving, this result does not fit with a strict interpretation of encoding specificity. However, the alphabetic-none condition did a large amount of alphabetic clustering on their first recall test (.81), which was even more than the alphabetic-letter condition on the uncued recall (.54). The negative correlation obtained between alphabetic clustering and recall may account for the comparatively low performance by the alphabetic-none group. (See also Lauer \& Battig, 1972).

The breakdown of cued recall into taxonomic NC and IPC indicated that output cue was the critical factor in categories recalled, while IPC was determined mainly by type of input or encoding, in agreement with previous results (Mondani, Pellegrino, \& Battig, 1973). In the letter NC analysis, the alphabetic input condition recalled significantly fewer letter categories with uncued output (5.67) than with letter cues for output (6.71). The categoric input condition, however, recalled only insignificantly $(p>.10)$ fewer taxonomic categories with no cues (6.43) than with taxonomic cues (7.29). This suggests that the presence of cues at output was more important for the recall of the alphabetic than the categoric encoding and organization.

\section{REFERENCES}

Battig, W. F., \& Montague, W. E. Category norms for verbal items in 56 categories: A replication and extension of the Connecticut category norms. Journal of Experimental Psychology Monograph, 1969, 80, No. 3, Part 2.

Lauer, P. A., \& Battig, W. F. Free recall of taxonomically and alphabetically organized word lists as a function of storage and retrieval cues. Journal of Verbal Learning \& Verbal Behavior, 1972, 11, 333-342.

Mondani, M. S., Pellegrino, J. W., \& Battig, W. F. Free and cued recall as a function of different levels of word processing. Journal of Experimental Psychology, 1973, 101, 324-329.

'Thomson, D. M., \& Tulving, E. Associative encoding and retrieval: Weak and strong cues. Journal of Experimental Psychology, 1970, 86, 255-262.

(Received for publication (June 12, 1974.) 